\title{
GBP-Monitor: Einschätzungen und Erwartungen deutscher Unternehmer angesichts des Regierungswechsels
}

\author{
Jannis Bischof | Philipp Dörrenberg | Davud Rostam-Afschar | \\ Thomas Simon | Dirk Simons |Johannes Voget*
}

Seit dem Juli 2020 befragt das German Business Panel (GBP) deutsche Unternehmen regelmäßig zu betriebswirtschaftlich relevanten Themen mit Fokus auf die Bereiche des externen und internen Rechnungswesens sowie der Besteuerung.

\section{Deutliche Verbesserung der betriebswirtschaftlichen Situation seit dem Frühjahr 2021}

Während die Gewinne und Umsätze deutscher Unternehmen im Februar 2021 im Vergleich zum Vorjahresmonat noch durchschnittlich um mehr als $20 \%$ zurückgingen, haben sich diese im Laufe des Sommers spürbar erholt. Obwohl die Gewinnveränderung weiterhin hinter der Umsatzentwicklung $(+7,66 \%$ im Vergleich zum Vorjahresmonat) zurückbleibt, liegt auch diese im Oktober 2021 zum ersten Mal seit Ausbruch der Pandemie $(+1,69 \%)$ auf Wachstumskurs. Trotz aktueller Probleme durch hohe

\footnotetext{
Prof. Dr. Jannis Bischof | Lehrstuhl für ABWL und Unternehmensrechnung | Universität Mannheim Ijbischof@uni-mannheim.de

Prof. Dr. Philipp Dörrenberg | Lehrstuhl für ABWL und Betriebswirtschaftliche Steuerlehre | Universität Mannheim

Dr. Davud Rostam-Afschar | Akademischer Leiter des German Business Panels | Universität Mannheim

Thomas Simon | Lehrstuhl für ABWL und Rechnungswesen I Universität Mannheim Prof. Dr. Dirk Simons | Lehrstuhl für ABWL und Rechnungswesen | Universität Mannheim Prof. Dr. Johannes Voget | Lehrstuhl für ABWL, Taxation \& Finance | Universität Mannheim
} 
Energiepreise und weitläufige Lieferengpässe durchbricht dieses positive Signal den langanhaltenden Rückgang der Unternehmensgewinne.

\section{Weiterhin hohe Unsicherheit bei zunehmend dynamischerem Pandemieverlauf}

Die lange Phase des wirtschaftlichen Abschwungs während der Pandemie schlägt sich auch spürbar in den unternehmerischen Entscheidungen nieder: Mitten in der vierten Corona-Welle treffen die Unternehmen vermehrt bestands- und liquiditätssichernde Maßnahmen, indem sie überwiegend Fixkosten reduzieren und mehrheitlich Preise erhöhen anstatt senken wollen. Die Trendwende bei den Unternehmensgewinnen wird daher vor allem durch Kostensenkungen und Preiserhöhungen und nicht durch Nachfrageeffekte erklärt.

Einige dieser Reaktionen auf die Krise gehen zu Lasten der Zukunft, insbesondere wenn Unternehmen nun vermehrt Aufwendungen für Forschung und Entwicklung kürzen. Die hohe Unsicherheit zeigt sich zudem in einem erheblichen Risiko von Unternehmensschließungen: Der Anteil an Unternehmen, die nach Einschätzung von Wettbewerbern aus derselben Branche innerhalb des kommenden Jahres aus der Geschäftstätigkeit ausscheiden werden, liegt im Oktober 2021 im Mittel noch bei 12,7\%.

\section{Verhaltene Zufriedenheit mit der Wirtschafts- und Corona-Politik der alten Bundesregierung}

In Bezug auf die allgemeine Wirtschaftspolitik der aktuell geschäftsführenden Bundesregierung äußern sich die deutschen Unternehmer und Manager verhalten. So liegt die allgemeine Zufriedenheit mit der Wirtschaftspolitik im Herbst konstant nur knapp über 4 Punkten auf einer 10Punkte Skala. Obgleich speziell die Corona-Politik mit 4,8 Punkten im Mittel etwas besser beurteilt wird, gibt die GBP-Unternehmensstudie Aufschluss darüber, welche wirtschaftlichen Folgen der Pandemie speziell der Politik zur Last gelegt werden. Während die Unternehmen der Politik nämlich die Verpflichtung zu Geschäftsschließungen sowie das Fehlen von Mitarbeitern aufgrund von Schul- und Kindergartenschließungen negativ zuschreiben, werden andere Krisenmaßnahmen wie Home-OfficeVerpflichtungen und Digitalisierungszwang auch dann nicht negativ beurteilt, wenn sie mit finanziellen Belastungen einhergehen.

\section{Forderung nach Maßnahmen gegen Fachkräftemangel und für Digitalisierung an zukünftige Koalition}

Als zukünftige Bundesregierung präferieren seit der Bundestagswahl am 26. September $2021 \mathrm{mehr}$ als $50 \%$ der Unternehmen eine Ampel-Koalition von SPD, Bündnis 90/Die Grünen und FDP, während eine Jamaika-Koali- 
tion unter Beteiligung der CDU lediglich von einem Viertel der Manager gewünscht wird. Von der zukünftigen Bundesregierung erwarten die Unternehmer vor allem Maßnahmen gegen den Fachkräftemangel sowie eine Digitalisierungsoffensive. Insbesondere halten deutsche Unternehmer diese Themen für wichtiger als Steuersenkungen. Zusätzlich bekräftigen die Befragungsergebnisse des GBP die Notwendigkeit einer Ausweitung des Klimaschutzes: Demnach halten beinahe $20 \%$ der befragten Manager und Unternehmer die Umsetzung zusätzlicher klimapolitischer Maßnahmen für das drängendste wirtschaftspolitische Thema, während einer Erhöhung des Mindestlohns und dem Abbau von Staatsschulden nur geringe Relevanz beigemessen wird.

\section{Quelle}

Die Meldung basiert auf „Betriebswirtschaftliche Einschätzungen und Erwartungen von Unternehmen in Deutschland. GBP-Monitor: Unternehmenstrends im September 2021. TRR 266 Accounting for Transparency. https://doi.org/10.52569/QRPQ4370.", „Betriebswirtschaftliche Einschätzungen und Erwartungen von Unternehmen in Deutschland. GBP-Monitor: Unternehmenstrends im Oktober 2021. TRR 266 Accounting for Transparency. https://doi.org/10.52569/ZCYA6673.“ sowie „Betriebswirtschaftliche Einschätzungen und Erwartungen von Unternehmen in Deutschland. GBP-Monitor: Unternehmenstrends im November 2021. TRR 266 Accounting for Transparency. https://doi.org/10.52569/KRAX2859.“ von Jannis Bischof, Philipp Dörrenberg, Davud Rostam-Afschar, Dirk Simons und Johannes Voget. 